\title{
APC downregulated 1 inhibits breast cancer cell invasion by inhibiting the canonical WNT signaling pathway
}

\author{
SUNG-GOOK CHO
}

\begin{abstract}
Department of Biotechnology, Korea National University of Transportation, Jeungpyeong, Chungbuk 368-701, Republic of Korea
\end{abstract}

Received September 19, 2016; Accepted June 9, 2017

DOI: $10.3892 / \mathrm{ol} .2017 .6801$

\begin{abstract}
Canonical WNT signaling promotes breast cancer progression. Although APC downregulated 1 (APCDD1) may inhibit canonical WNT signaling, its role in breast cancer remains to be fully understood. The present study demonstrated that APCDD1 suppressed in vitro breast cancer growth and metastasis by inhibiting canonical WNT signaling. The present study demonstrated that APCDD1 expression was negatively associated with breast cancer cell invasion, which was consistent with previous studies that indicated that APCDD1 expression was decreased in invasive ductal carcinoma compared with that in ductal carcinoma in situ. Furthermore, APCDD1 expression was negatively associated with nuclear $\beta$-catenin expression and transcription factor/lymphoid enhancer binding factor 1 transcriptional activity in the present study. Silencing APCDD1 in non-invasive breast cancer cells using lentiviral APCDD1 short hairpin RNAs enhanced migration and invasion, which may be mediated by canonical WNT signaling, whereas the overexpression of human influenza hemagglutinin-tagged APCDD1 in invasive breast cancer cells repressed these properties. Therefore, the present study suggested that APCDD1 regulated breast cancer progression by targeting canonical WNT signaling and modulating breast cancer cell invasion.
\end{abstract}

\section{Introduction}

The WNT signaling pathway is associated with numerous biological events, including embryonic development and adult tissue homeostasis. Therefore, abnormal WNT signaling is associated with diseases, including certain types of cancer $(1,2)$. WNTs activate the canonical and non-canonical

Correspondence to: Dr Sung-Gook Cho, Department of Biotechnology, Korea National University of Transportation, 61 University Road, Jeungpyeong, Chungbuk 368-701, Republic of Korea

E-mail: chosg@ut.ac.kr

Key words: APC downregulated 1, canonical WNT signaling, breast cancer signaling pathways, which are mutually exclusive. The canonical WNT signaling pathway triggers $\beta$-catenin-dependent transcriptional regulation, whereas the non-canonical WNT signaling pathway activates the $\beta$-catenin-independent signaling pathway $(1,2)$.

The canonical WNT signaling pathway regulates normal breast development, and its deregulation is associated with breast cancer progression $(3,4)$. WNTs aid in the generation of the canonical WNT signaling pathway by binding to the coreceptors LDL receptor-related protein (LRP)5/6 and frizzled on the cell surface, and activating the $\beta$-catenin/t-cell factor (TCF) complex (2). Overexpression of Wnt1, which may be induced by the integration of the mouse mammary tumor virus, triggers mammary tumor development $(5,6)$. Autocrine WNT signaling regulates mammary epithelial cell fate by regulating renewal and the epithelial-to-mesenchymal transition (EMT), thereby affecting tumorigenesis and metastasis in a deregulated signaling state $(7,8)$. Accordingly, the WNT-activated $\beta$-catenin/TCF complex potentiates breast cancer metastasis by altering the expression of genes associated with EMT $(9,10)$.

Previous studies have revealed multiple inhibitors of the canonical WNT signaling pathway, including secreted frizzled-related protein (SFRP), dickkopf (DKK), and WNT inhibitory factor (WIF) $(2,11)$. In addition, APC downregulated 1 (APCDD1) may inhibit the canonical WNT signaling pathway by directly binding WNT3A and LRP5 on the cell surface $(11,12)$. Therefore, APCDD1 represents an inhibitor of the canonical WNT signaling pathway. While a previous study demonstrated that APCDD1 promoted colorectal cancer growth, its function as the inhibitor of the canonical WNT signaling pathway was not assessed (13). Furthermore, its function in different types of cancer, including breast cancer, remains to be fully understood. The present study evaluated the function of APCDD1 in breast cancer cells and revealed that APCDD1 regulated breast cancer cell invasion.

\section{Materials and methods}

Cell culture, reagents and plasmids. The HEK-293T, non-invasive breast cancer MCF-7 and T-47D, invasive breast cancer SKBR3 and MDA-MB-231 cell lines were cultured in Dulbecco's modified Eagle's medium supplemented with $10 \%$ fetal bovine serum (FBS; Gibco; Thermo Fisher Scientific, Inc., 
Waltham, MA, USA) and $1 \%$ penicillin/streptomycin at $37^{\circ} \mathrm{C}$ in a humidified atmosphere of $5 \% \mathrm{CO}_{2}$ and $95 \%$ air. Invasive breast cancer HCC-1419 and HCC-70 cell lines were cultured in RPMI-1640 medium (Gibco; Thermo Fisher Scientific, Inc.) supplemented with $10 \%$ FBS and $1 \%$ penicillin/streptomycin at $37^{\circ} \mathrm{C}$ in a humidified atmosphere of $5 \% \mathrm{CO}_{2}$ and $95 \%$ air. A concentration of $1 \times 10^{6}$ MCF-7 or MDA-MB-231 cells were transfected with the appropriate plasmids for $24 \mathrm{~h}$ using Lipofectamine 2000 (Invitrogen; Thermo Fisher Scientific, Inc.), according to the manufacturer's protocol, and was cultured for another $24 \mathrm{~h}$ at $37^{\circ} \mathrm{C}$. Recombinant human WNT3A (catalog no. 5036-GMP; recombinant human WNT3A GMP, carrier free) was purchased from R\&D Systems, Inc. (Minneapolis, MN, USA). To assess the effect of WNT3A, MCF-7 and MDA-MB-231 cells were treated with $1 \mu \mathrm{g} / \mathrm{ml}$ recombinant human WNT3A. Cyclosporine A, FK506 and BAPTA-AM were obtained from Tocris Bioscience (Bristol, UK). EGTA-AM was purchased from EMD Millipore (Billerica, MA, USA). Lentiviral APCDD1 short hairpin (sh)RNAs [1, TRCN0000413419 (CCGGGAAAGCTAGGGCCTCTT ATTTCTCGAGAAATAAGAGGCCCTAGCTTTCTTTTT TG); 2, TRCN0000136710 (CCGGGAGCTCTTCCTTGG TGACATTCTCGAGAATGTCACCAAGGAAGAGCTCTT TTTTG); 3, TRCN0000136596 (CCGGCGGTGCACAAA TCCCACTTATCTCGAGATAAGTGGGATTTGTGCACC GTTTTTTG); 4, TRCN0000137317 (CCGGGCCAGAGA ACTGTCCTTCTTTCTCGAGAAAGAAGGACAGTTCTC TGGCTTTTTTG); and 5,TRCN0000136748(CCGGGCTGG AATCCAATGCAGAGTTCTCGAGAACTCTGCATTGGA TTCCAGCTTTTTTG)] and control plasmids pLKO TRC005 (TRCN0000231701; target sequence TCAGTTCCAGTACGG CTCCAA) and pLKO TRC001 (TRCN0000072208; target sequence GCTTCAAGTGGGAGCGCGTGA) were obtained from Sigma-Aldrich; Merck KGaA (Darmstadt, Germany). pMD2.G (12259; Addgene, Inc., Cambridge, MA, USA) and psPAX2 (12260; Addgene, Inc.) were used for lentiviral packaging. A full length HindIII/XhoI fragment of APCDD1 was inserted into a pCMV6-AC-HA plasmid(Origene Technologies, Inc., Rockville, MD, USA). For the APCDD1-luciferase (luc) plasmid, the APCDD1 promoter regions between -1,000 bp and $+1 \mathrm{bp},-500 \mathrm{bp}$ and $+1 \mathrm{bp}$, and $-200 \mathrm{bp}$ and +1 bp were inserted into a pGL3-Basic vector (Promega Corporation, Madison, WI, USA). Wild-type $\beta$-catenin ( $\beta$-catenin-WT; pcDNA3- $\beta$-catenin; Addgene ID, 16828), $\beta$-catenin-S33Y (pcDNA3-S33Y- $\beta$-catenin; Addgene ID, 19286), $\beta$-catenin shRNA (pLKO.1 puro shRNA $\beta$-catenin; Addgene ID, 18803), TCF4 mutant lacking $\beta$-catenin interaction domain $(\triangle \mathrm{N}-\mathrm{TCF} 4$; pcDNA/MYC proto-oncogene- $\Delta$ N-TCF4; Addgene ID, 16513) and TCF/lymphoid enhancer binding factor (LEF) reporter plasmids (M50 Super 8x TOPFlash, Addgene ID, 12456; M51 Super 8x FOPFlash, Addgene ID, 12457) were obtained from Addgene, Inc. (Cambridge, MA, USA). All experiments were performed at least three times.

Chromatin immunoprecipitation (ChIP) and luc assays. TCF4 binding sites at $-1,000 \mathrm{bp}$ upstream of the APCDD1 gene were analyzed in silico using the publicly available programs PROMO, LASAGNA and JASPAR (14-17). ChIP assays using a ChIP kit (Abcam, Cambridge, MA, USA) were performed according to the manufacturer's protocol. A concentration of
$3 \times 10^{6}$ MCF-7 and MDA-MB-231 cells were subjected to the ChIP assays. As a negative control, rabbit IgG (Abcam) was used. Quantitative polymerase chain reaction (qPCR) was performed using SYBR Green real-time PCR master mix (cat no. 4309,155; Thermo Fisher Scientific, Inc.), according to the manufacturer's protocol. Samples were incubated for $10 \mathrm{~min}$ at $95^{\circ} \mathrm{C}$, denatured for $15 \mathrm{sec}$ at $95^{\circ} \mathrm{C}$ and annealed and extended for $1 \mathrm{~min}$ at $60^{\circ} \mathrm{C}$, for 40 cycles. qPCR reactions were performed using a LightCycler 480 Instrument II (Roche Diagnostics, Indianapolis, IN, USA), and relative quantifications were automatically performed using LightCycler 480 software 1.5 (Roche Diagnostics). Primer sequences for ChiP assays were as follows: forward 5'-TTGGGTCTCAAACGC CCATG-3', reverse 5'-TTCATATTTCCAGCGCGCGCC-3'. $G A P D H$ was used as a positive control. Its primer sequences are as follows: Forward, 5'-CGGGATTGTCTGCCCTAATTA T-3' and reverse, 5'-GCACGGAAGGTCACGATGT-3' (18). The reporter plasmid pAPCDD1-luc was subjected to the luc assay. Luc assays were performed using a Dual-Luciferase Reporter Assay system (Promega, Madison, WI, USA). In brief, activities of firefly and Renilla luciferases were sequentially determined and firefly luciferase activity was divided by Renilla luciferase activity to obtain a fold activity. All experiments were performed in triplicate and independently repeated three times.

Western blot analysis and immunoprecipitation assays. Antibodies against actin (sc-47778; dilution, 1:200) and lamin (sc-6215; dilution, 1:200) were purchased from Santa Cruz Biotechnology, Inc. (Dallas, TX, USA). Antibodies against TCF4 (dilution, 1:1,000; 2569), $\beta$-catenin (dilution, 1:1,000; 8480), snail (dilution, 1:1,000; 3879), twist (dilution 1:1,000; 46702), vimentin (dilution, $1: 1,000 ; 5741$ ), epithelial (E) -cadherin (dilution, 1:1,000; 3195) and neural $(\mathrm{N})$-cadherin (dilution, 1:1,000; 13116) were purchased from Cell Signaling Technology, Inc. (Danvers, MA, USA). Antibodies against APCDD1 (dilution, 1:1,000; ab73063) and hemagglutinin (HA) were purchased from Abcam. Horseradish peroxidase-conjugated anti-rabbit IgG (\#7074) or anti-mouse IgG (\#7076) antibodies (Cell Signaling Technology, Inc.) were used as secondary antibodies at a dilution of 1:10,000. Nuclear fraction was achieved using a Cell Fractionation kit (Cell Signaling Technology, Inc.). Immunoprecipitation assays were performed using protein A/G Plus agarose beads (Santa Cruz Biotechnology, Inc.). For western blot analysis, $3 \times 10^{6}$ cells (all cell types analyzed in this study) were lyzed using radioimmunoprecipitation buffer for $30 \mathrm{~min}$ on ice and centrifuged at $20,000 \mathrm{x} \mathrm{g}$ for $10 \mathrm{~min}$ at $4^{\circ} \mathrm{C}$. Evaluation of protein concentrations was performed using Pierce BCA protein assay kit (Pierce; Thermo Fisher Scientific, Inc.), according to the manufacture's protocol. A total of $30 \mu \mathrm{g}$ protein/lane was loaded to $10-12 \%$ SDS-PAGE and transferred to a polyvinylidene difluoride membrane (GE Healthcare Life Sciences, Little Chalfont, UK). For the immunoprecipitation assays, $300 \mu \mathrm{g}$ of nuclear protein from either MCF-7 or MDA-MB-231 cells, $10 \mu \mathrm{l}$ protein A/G plus agarose solution $(0.5 \mathrm{ml}$ agarose $/ 2.0 \mathrm{ml}$ solution; Santa Cruz Biotechnology, Inc.) and $1 \mu \mathrm{g}$ appropriate antibody were mixed and incubated for $12 \mathrm{~h}$ at $4^{\circ} \mathrm{C}$. Subsequent to blocking with $5 \%$ milk for $1 \mathrm{~h}$ at room temperature, the membrane was incubated with the aforementioned antibodies for another $1 \mathrm{~h}$ 
A

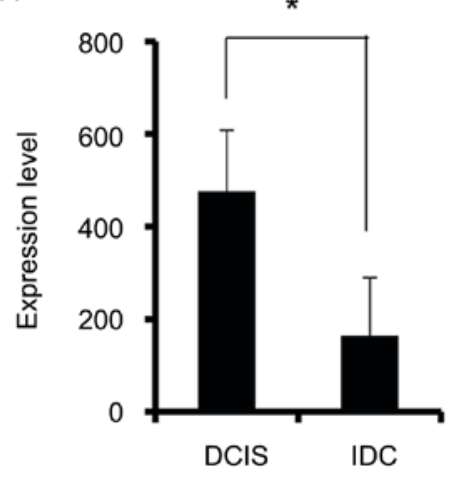

B

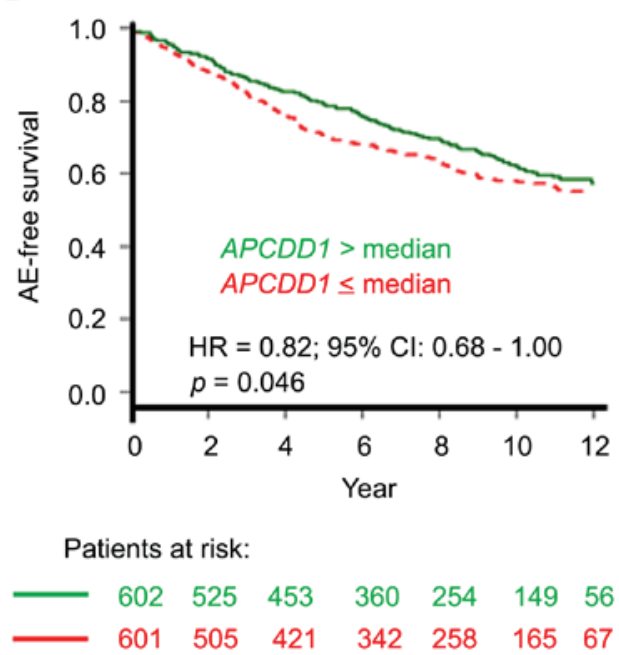

Figure 1. APCDD1 expression in breast cancer. (A) Expression of APCDD1 in DCIS and IDC. Data for the expression of APCDD1 were obtained from the Gene Expression Omnibus GSE21422 dataset. (B) APCDD1 expression in GSE1456 and GSE10510 indicated that increased APCDD1 expression was associated with a more favorable prognosis in terms of any event-free survival. DCIS, ductal carcinoma in situ; IDC, invasive ductal carcinoma; AE, any event; HR, hazard ratio; CI, confidence interval; APCDD1, APC downregulated 1.

at room temperature. Actin was detected as an internal control. The membranes were then incubated with the aforementioned secondary antibodies for $1 \mathrm{~h}$ at room temperature. Western bands were detected using LumiGLO chemiluminescent reagent and peroxidase (\#7003; Cell Signaling Technology Inc.). Western blot analyses were performed independently three times. ImageJ software (version 1.50) was used for relative quantifications (National Institutes of Health, Bethesda, MA, USA).

Cell proliferation, migration and invasion assays. A concentration of $3 \times 10^{5}$ APCDD1-overexpressing or APCDD1-silenced cells together with the control cells were separately cultured in 6-well plates for $72 \mathrm{~h}$ at $37^{\circ} \mathrm{C}$, and cell numbers were counted every day. Experiments were performed in quadruplicate and independently repeated in triplicate. For cell migration, $3 \times 10^{5}$ APCDD1-overexpressing or APCDD1-silenced cells together with the control cells were cultured in 6-well plates and scratched at $37^{\circ} \mathrm{C}$ when the confluence reached $\sim 80 \%$, and then the number of migrated cells was counted $24 \mathrm{~h}$ after scratching. Experiments were performed in triplicate. For the invasion assays, $3 \times 10^{5}$ APCDD1-overexpressing or APCDD1-silenced cells together with the control cells were cultured in the upper chambers of Matrigel-pre-coated Transwell plates and incubated for $16 \mathrm{~h}$ at $37^{\circ} \mathrm{C}$. The cells in the upper chamber were removed using a swab and the cells that had invaded through the Matrigel were stained with $0.4 \%$ crystal violet for $10 \mathrm{~min}$ at room temperature, washed with water and then counted. Experiments were performed in triplicate. The cell migration and invasions were determined using a Zeizz Axiovert inverted microscope, and the images were analyzed using Zen software version 3.00 (Carl Zeizz, Oberkochen, Germany). A total of 4 fields were randomly selected and the migrated or invaded cells were counted.

Data mining from gene expression dataset. From the Gene Expression Omnibus (GEO) datasets in NCBI (https://www. ncbi.nlm.nih.gov/geo/), GSE21422 series was chosen as it featured gene expression profiles of human ductal carcinoma in situ (DCIS) and invasive ductal breast carcinoma (IDC). APCDD1 expression levels in DCIS and IDC were then analyzed using data analysis tools for GDS3853 in dataset browser. Any event (AE)-free event plot with APCDD1 expression pattern was obtained from the website, Breast Cancer Gene-Expression Miner v4.0 (http://bcgenex. centregauducheau.fr).

Statistical analysis. Unpaired Student's t-test or one-way analysis of variance with a post-hoc Tukey's test was performed to calculate the statistical significance of the results of the present study. Results were presented as the mean \pm standard deviation and $\mathrm{P}<0.05$ was considered to indicate a statistically significant difference. Calculations were performed using SPSS version 22 (IBM Corp., Armonk, NY, USA) software.

\section{Results}

APCDD1 expression pattern in breast cancer. The Gene Expression Omnibus GSE21422 dataset $(19,20)$ revealed that the level of APCDD1 transcript was significantly increased in ductal carcinoma in situ (DCIS) compared with that in invasive ductal carcinoma (IDC) (Fig. 1A). Furthermore, the GSE1456 and GSE10510 datasets on patients with breast cancer (20-22) demonstrated that increased APCDD1 expression was associated with a favorable prognosis in terms of any event-free survival (Fig. 1B). These datasets suggested that APCDD1 may negatively regulate breast cancer progression.

APCDD1 expression pattern in breast cancer cell lines. The expression patterns of APCDD1 in multiple breast cancer cell lines were assessed. Although WNT3A expression did not differ between the breast cancer cell lines, APCDD1 expression was increased in non-invasive compared with invasive breast cancer cells (Fig. 2A). This result was consistent with 


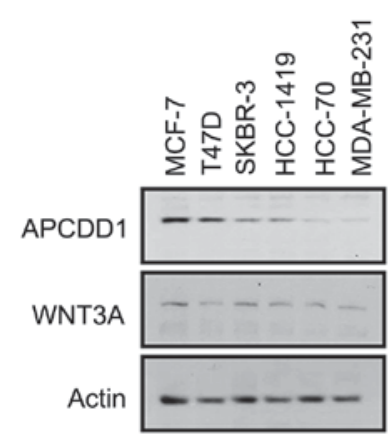

B

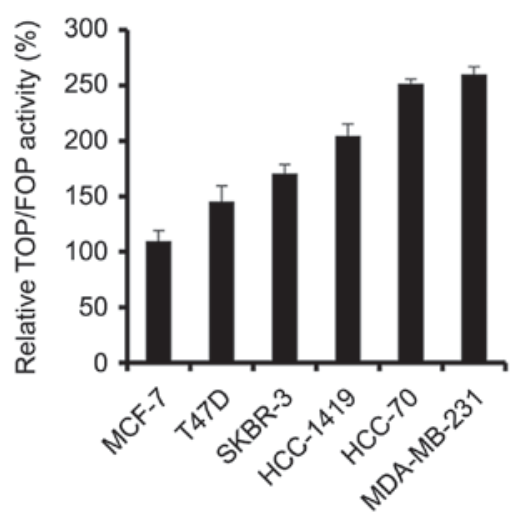

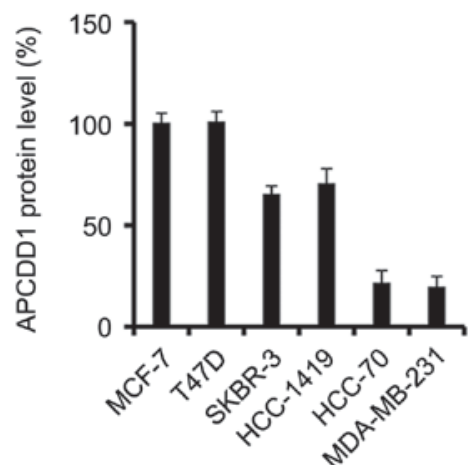

C

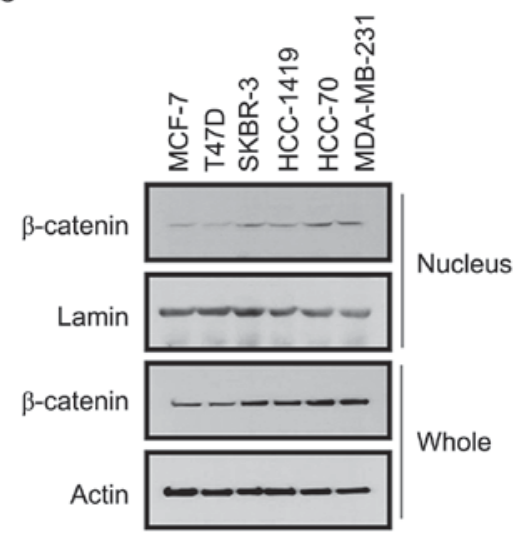

Figure 2. APCDD1 expression in multiple types of breast cancer cell. (A) Expression of APCDD1 and WNT3A in multiple types of breast cancer cell. Actin was used as a loading control. (B) Transcription factor/lymphoid enhancer binding factor transcription in multiple types of breast cancer cell. The cells were transfected with TOPflash or FOPflash. The activity of TOPflash was normalized to that of FOPflash. (C) Expression of $\beta$-catenin in multiple types of breast cancer cell. Lamin was used as the internal control for nuclear protein. Actin was used as the internal control for whole protein. TOP, TOPflash; FOP, FOPflash; APCDD1, APC downregulated 1.

data where APCDD1 expression was decreased in IDC compared with that in DCIS (Fig. 1A).

As APCDD1 inhibits the canonical WNT signaling pathway (12), TCF/LEF transcription was assessed in the breast cancer cells. TCF/LEF transcription was positively associated with the invasive ability of the breast cancer cells (Fig. 2B), suggesting a negative association between TCF/LEF transcription and APCDD1 expression.

Since $\beta$-catenin is crucial for TCF/LEF transcription $(23,24), \beta$-catenin expression in the breast cancer cells was assessed. The invasive breast cancer cells exhibited increased expression of nuclear and total $\beta$-catenin compared with that exhibited by the non-invasive breast cancer cells (Fig. 2C). Therefore, the results of the present study demonstrated that the canonical WNT signaling pathway is positively associated with invasion in breast cancer cells, which suggests that APCDD1 may regulate breast cancer cell invasion, as driven by the canonical WNT signaling pathway.

APCDD1 function in the canonical WNT signaling pathway in breast cancer cells. Since APCDD1 expression was associated with the invasive phenotype of breast cancer cells, the function of APCDD1 in the invasion of breast cancer cells was assessed. Constructs 2 and 3 of the lentiviral APCDD1 shRNA plasmids repressed APCDD1 expression in MCF-7 cells
(Fig. 3A). Hemagglutinin (HA)-tagged APCDD1 was overexpressed in MDA-MB-231 cells and detected using an antibody for either HA or APCDD1 (Fig. 3A). Altered APCDD1 expression negatively affected nuclear $\beta$-catenin expression (Fig. 3B) and TCF/LEF functions (Fig. 3C). APCDD1 function in breast cancer cell metastasis. Since the canonical WNT signaling pathway regulates the expression of EMT-associated genes and promotes invasion in breast cancer cells (9), the present study assessed whether altering APCDD1 expression in breast cancer cells affects the expression patterns of EMT-associated genes. Silencing APCDD1 in MCF-7 cells or inducing APCDD1 overexpression in MDA-MB-231 cells altered the expression of EMT-associated genes, including zinc finger E-box binding homeobox 1 (Zeb1), Twist, Vimentin, E-cadherin and $\mathrm{N}$-cadherin, while altering APCDD1 expression did not affect snail expression (Fig. 4A). APCDD1 silencing increased the expression levels of Zeb1, Twist, Vimentin and N-cadherin and reduced E-cadherin expression level. APCDD1 overexpression reversed expression patterns of those proteins.

Furthermore, silencing APCDD1 in MCF-7 cells promoted migration and invasion, and its overexpression in MDA-MB-231 cells attenuated migration and invasion (Fig. 4B and C). However, altering APCDD1 expression did not significantly affect growth in these cell lines (data not shown). Therefore, these results suggested that APCDD1 inhibited the invasion of the breast cancer cells. 
A
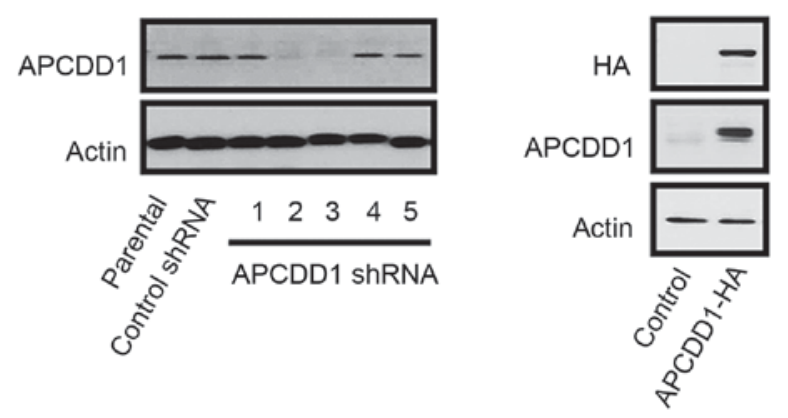

B

C
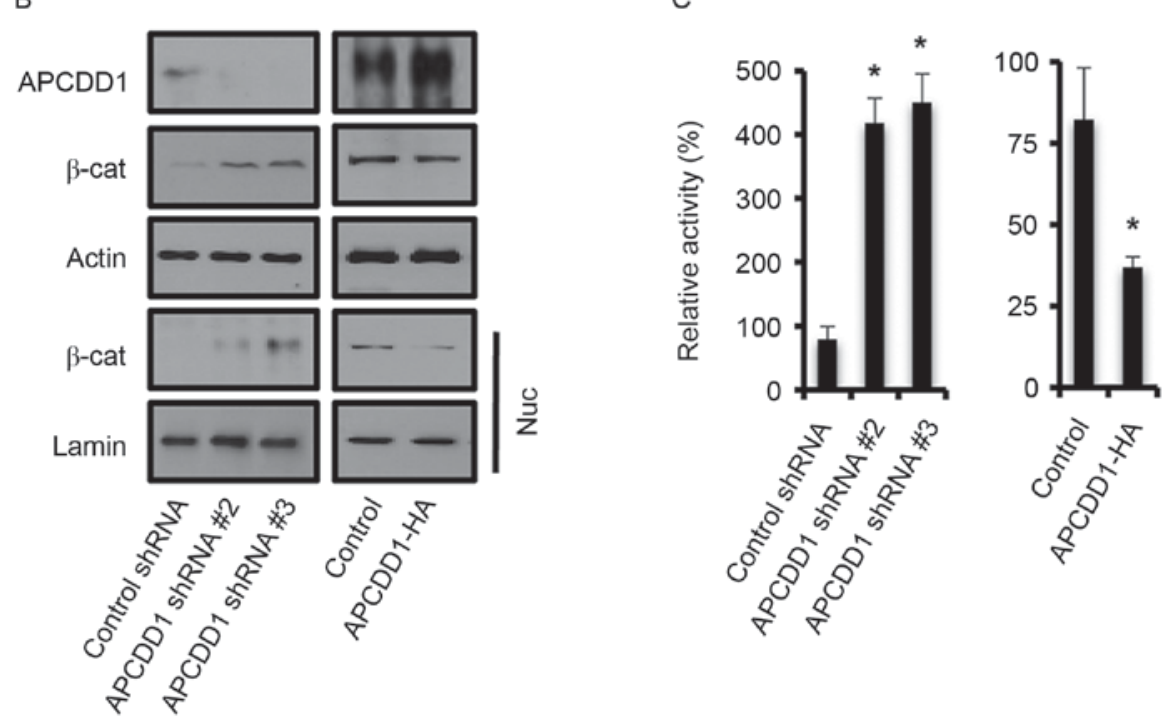

Figure 3. APCDD1 negatively regulates the canonical WNT signaling pathway. (A) APCDD1 silencing or overexpression was confirmed using western blot analysis. MCF-7 cells were infected with lentiviral APCDD1 shRNA or scramble shRNA (as a control). MDA-MB-231 cells were transfected with APCDD1 or empty vector. (B) APCDD1 altered nuclear $\beta$-catenin expression. APCDD1 was silenced or overexpressed in MCF-7 and MDA-MB-231 breast cancer cells, respectively, and nuclear $\beta$-catenin was subsequently detected in the nuclear fractions. (C) Transcription factor/lymphoid enhancer binding factor transcription activity was measured using TOP/FOP reporter assays. Experiments were performed in triplicate and independently repeated three times. ${ }^{*} \mathrm{P}<0.05$ vs. the control group. sh, short hairpin; $\beta$-cat, $\beta$-catenin; HA, hemagglutinin; APCDD1, APC downregulated 1.

Canonical WNT signaling pathway regulates APCDDI expression via the TCF4/ $\beta$-catenin complex. Since $\beta$-catenin is crucial for TCF4 transcription (23-25), $\beta$-catenin interaction with TCF4 in the breast cancer cells was further assessed. The interaction rate between $\beta$-catenin and TCF4 was increased in highly invasive MDA-MB-231 cells compared with that in less invasive MCF-7 cells (Fig. 5A). Subsequently, TCF4 interaction with APCDD1 promoter regions was evaluated in $\mathrm{MCF}-7$ and MDA-MB-231 breast cancer cell lines using ChIP assays with anti-TCF4 antibodies. The interaction rate of TCF4 with APCDD1 promoter regions was increased in MDA-MB-231 cells compared with that in MCF-7 cells (Fig. 5B). Therefore, the results of the present study suggested that the canonical WNT signaling pathway was more active in the invasive than in the non-invasive breast cancer cells.

The results of the present study revealed that APCDD1 expression was negatively associated with $\mathrm{TCF} 4 / \beta$-catenin activity. Therefore, the present study assessed how the $\beta$-catenin/TCF4 complex regulates APCDD1 promoter activity. In MCF-7 cells, the recombinant human WNT3A significantly increased APCDD1 promoter activity $6 \mathrm{~h}$ after treatment, but reduced it again $12 \mathrm{~h}$ after treatment (Fig. 5C). However, WNT3A did not significantly increase APCDD1 promoter activity in MDA-MB-231 cells at any time after treatment (Fig. 5C).
The present study found that, following the transfection of MCF-7 cells with $\triangle \mathrm{N}-\mathrm{TCF} 4$ and treatment with WNT3A for $6 \mathrm{~h}$, APCDD1 promoter activity was not altered as compared with the control (Fig. 5D), indicating that WNT3A requires TCF4 for APCDD1 promoter activation.

The present study demonstrated that the canonical WNT signaling pathway positively regulated APCDD1 expression in the non-invasive breast cancer cells, whereas the activity of the canonical WNT signaling pathway was negatively associated with APCDD1 expression. Therefore, this suggested that APCDD1 repressed the induction of APCDD1 expression by WNT3A through a negative feedback mechanism. Silencing APCDD1 in MCF-7 cells using lentiviral APCDD1 shRNA and subsequently treating the cells with WNT3A for $12 \mathrm{~h}$ increased APCDD1 promoter activity (Fig. 5E). Therefore, the results of the present study indicated that the canonical WNT signaling pathway in the non-invasive breast cancer cells generated APCDD1-mediated negative feedback signaling.

\section{Discussion}

The canonical WNT signaling pathway is crucial for breast cancer progression, including distant metastasis $(1,2,4,6)$. APCDD1 represents an inhibitor of the canonical WNT 


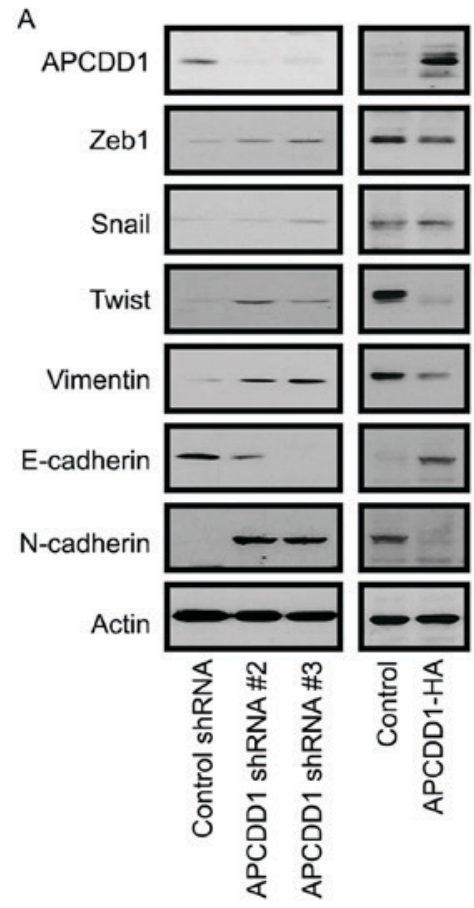

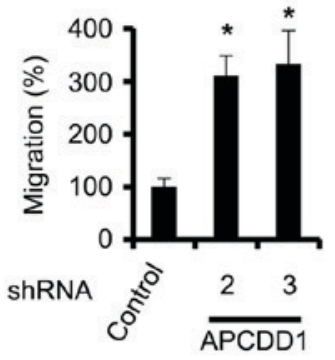

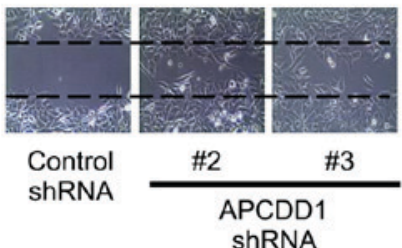

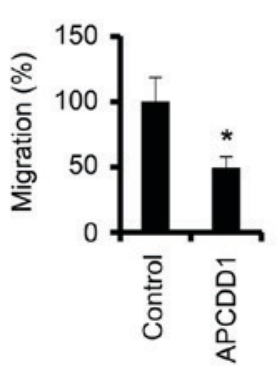

C
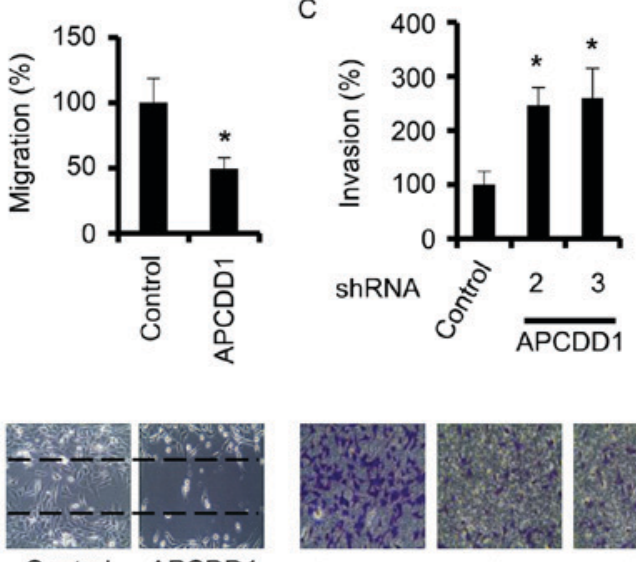

Control APCDD1

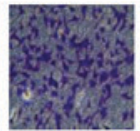

Control ShRNA
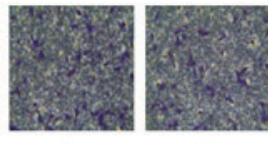

\#3

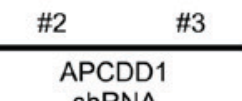

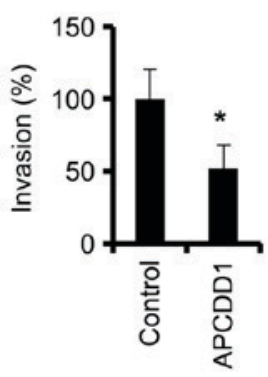
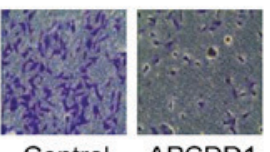

Control APCDD1

Figure 4. APCDD1 negatively regulates metastasis in breast cancer cells. (A) APCDD1 regulated the expression of EMT-associated proteins in breast cancer cells. The results of western blot analysis demonstrated the APCDD1-induced alterations to EMT-associated protein expression. (B) To assess cell migration, the cells were scratched and subsequently cultured for $24 \mathrm{~h}$. (C) To evaluate cell invasion, the cells were cultured on the top layer of the chamber, which was precoated with Matrigel, and incubated for $24 \mathrm{~h}$. Invasive cells were counted following the swabbing of the top layer. Cell migration and invasion assays were performed in triplicate and independently repeated three times. ${ }^{*} \mathrm{P}<0.05$ vs. the control group. EMT, epithelial-to-mesenchymal transition; Zeb1, zinc finger E-box binding homeobox 1; E, epithelial; N, neural; sh, short hairpin; APCDD1, APC downregulated 1.

signaling pathway (12). Although APCDD1 has been revealed to promote colorectal cancer cell proliferation (13), its functions in other types of cancer are yet to be fully understood. The present study demonstrated that APCDD1 suppressed the canonical WNT signaling pathway-driven invasion of breast cancer cells.

APCDD1 expression was decreased in the invasive compared with the non-invasive breast cancer cells. Furthermore, the results of the present study suggested that APCDD1, via its inhibitory function in the canonical WNT signaling pathway, suppressed the invasion of the breast cancer cells. When considering autocrine WNT signaling in breast cancer cells (8), APCDD1 may determine an input level of autocrine WNT signaling and thereby facilitate invasion.
The results of the present study demonstrated that the $\beta$-catenin/TCF4 complex regulated APCDD1 expression in the breast cancer cells, which is consistent with the results of a previous study that demonstrated that the $\beta$-catenin/TCF4 complex positively regulated APCDD1 expression in colorectal cancer cells (13). However, APCDD1 functioned as a negative regulator of the canonical WNT signaling pathway in the breast cancer cells of the present study, which is consistent with a previous study that revealed that APCDD1 binds to WNT3A and LRP5 to inhibit the canonical WNT signaling pathway in hair follicles (12). Although APCDD1 has been demonstrated to promote colorectal cancer cell proliferation (13), in the present study it suppressed breast cancer cell 

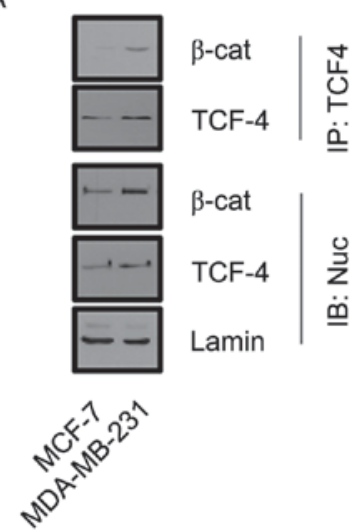

C

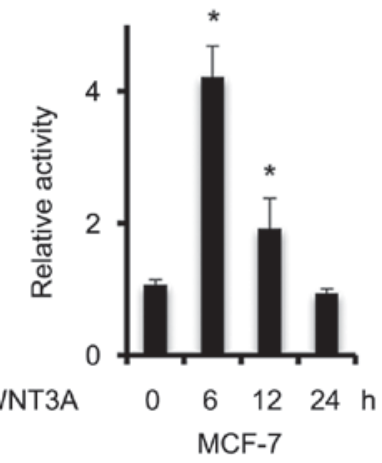

D

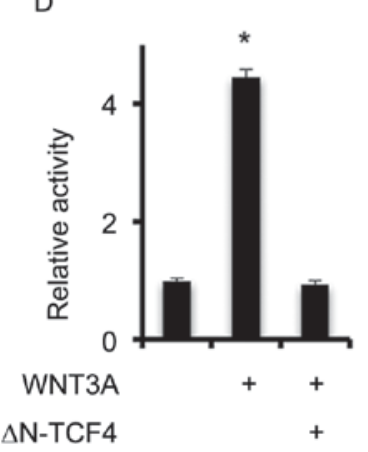

B

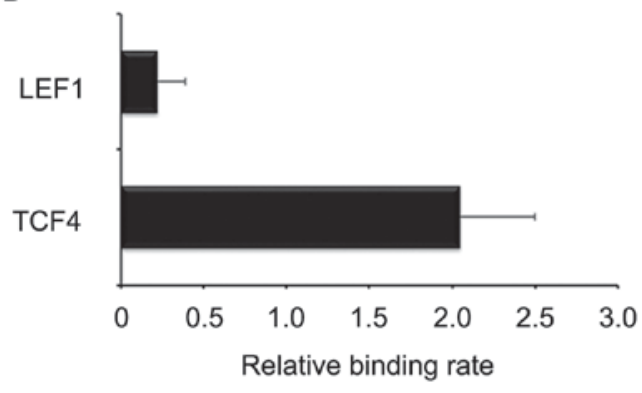

MDA-MB-231 vs. MCF-7

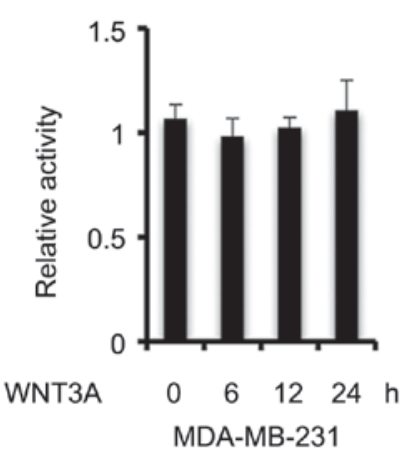

E

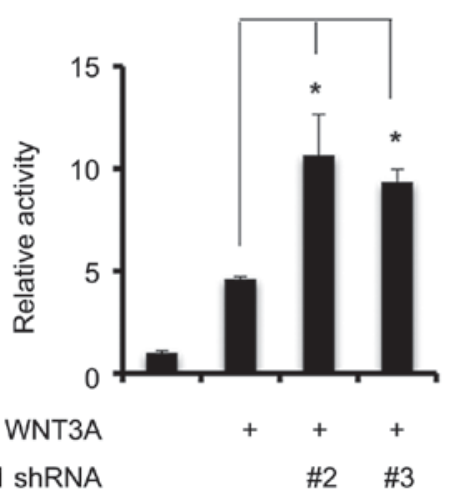

Figure 5. Canonical WNT signaling pathway regulates APCDD1 expression via the $\beta$-catenin/TCF4 complex. (A) Interaction between $\beta$-catenin and TCF4 in the MCF-7 and MDA-MB-231 breast cancer cells. Nuclear TCF4 was immunoprecipitated with an appropriate antibody and subsequently immunoblotted with the indicated antibodies. (B) TCF4 interaction with APCDD1 promoter regions in MCF-7 and MDA-MB-231 cells. TCF4 binding sites of the APCDD1 promoter region (between $-2,000$ and $+1 \mathrm{bp}$ ). Chromatin immunoprecipitation assays were performed using anti-LEF1 or anti-TCF4 antibodies. Interaction in the MCF-7 and MDA-MB-231 breast cancer cells was measured using quantitative polymerase chain reaction analysis. (C) APCDD1 promoter activity was measured using reporter assays. The cells were transfected with pAPCDD1-luc construct for $48 \mathrm{~h}$ and the medium was subsequently replaced with serum-depleted medium. The cells were treated with recombinant WNT3A for 6-24 h and luc activity was subsequently measured. (D) MCF-7 cells were transfected with pAPCDD1-luc and $\triangle \mathrm{N}$-TCF4 for $30 \mathrm{~h}$, with WNT3A for a further $6 \mathrm{~h}$, and subsequently subjected to luc assays. (E) MCF-7 cells were infected with lentiviral APCDD1 shRNA for $48 \mathrm{~h}$ and subsequently treated with WNT3A for a further $12 \mathrm{~h}$. Luc assays were then performed to measure APCDD1 promoter activity. Experiments were performed in triplicate and repeated independently three times. ${ }^{*} \mathrm{P}<0.05$ vs. the control. TCF, transcription factor; LEF, lymphoid enhancer binding factor; luc, luciferase; $\beta$-cat, $\beta$-catenin; $\triangle \mathrm{N}$-TCF4, TCF4 mutant lacking $\beta$-cat interaction domain; sh, short hairpin; APCDD1, APC downregulated 1.

invasion without affecting proliferation. Therefore, APCDD1 may balance the canonical WNT signaling pathway via the autocrine signaling pathway. However, the mechanisms that repress APCDD1 expression to potentiate the canonical WNT signaling pathway, which is crucial for invasion, since invasion depends on the altered expression of EMT-associated genes, is yet to be fully understood.

Inhibitors of WNT signaling pathways, including WIF, DKK and SFRP, have been demonstrated to repress breast cancer development and metastasis $(11,26,27)$. However, the manner in which WNT signaling pathways and their inhibitors function together during cancer progression remains unclear. Whereas APCDD1 expression is negatively associated with invasion, WIF, DKK and SFRP are epigenetically inactivated in breast cancer cells, independent of invasion $(28,29)$. Therefore, different WNT signaling pathway inhibitors may serve crucial functions in the multiple roles of the canonical WNT signaling pathway in breast cancer progression. In 
conclusion, inhibitors against WNT signaling pathway are numerous and their functions overlap. Thus, future research should investigate the specific roles of each inhibitor, which will be useful to design drugs and treatment schedules for breast cancer.

\section{Acknowledgements}

The present study was supported by the Korea National University of Transportation in 2016 and by the Basic Science Research Program through the National Research Foundation of Korea funded by the Ministry of Science, ICT and Future Planning (grant no. NRF-2014R1A1A1035831).

\section{References}

1. Clevers $\mathrm{H}$ and Nusse $\mathrm{R}: \mathrm{Wnt} / \beta$-catenin signaling and disease. Cell 149: 1192-1205, 2012.

2. Anastas JN and Moon RT: WNT signalling pathways as therapeutic targets in cancer. Nat Rev Cancer 13: 11-26, 2013.

3. Incassati A, Chandramouli A, Eelkema R and Cowin P: Key signaling nodes in mammary gland development and cancer: $\beta$-catenin. Breast Cancer Res 12: 213, 2010.

4. Smalley MJ and Dale TC: Wnt signalling in mammalian development and cancer. Cancer Metastasis Rev 18: 215-230, 1999.

5. Nusse R and Varmus HE: Many tumors induced by the mouse mammary tumor virus contain a provirus integrated in the same region of the host genome. Cell 31: 99-109, 1982

6. Li Y, Hively WP and Varmus HE: Use of MMTV-Wnt-1 transgenic mice for studying the genetic basis of breast cancer. Oncogene 19: 1002-1009, 2000.

7. Scheel C, Eaton EN, Li SH, Chaffer CL, Reinhardt F, Kah KJ, Bell G, Guo W, Rubin J, Richardson AL and Weinberg RA: Paracrine and autocrine signals induce and maintain mesenchymal and stem cell states in the breast. Cell 145: 926-940, 2011.

8. Bafico A, Liu G, Goldin L, Harris V and Aaronson SA: An autocrine mechanism for constitutive Wnt pathway activation in human cancer cells. Cancer Cell 6: 497-506, 2004.

9. Yook JI, Li XY, Ota I, Hu C, Kim HS, Kim NH, Cha SY, Ryu JK, Choi YJ, Kim J, et al: A Wnt-Axin2-GSK3beta cascade regulates Snaill activity in breast cancer cells. Nat Cell Biol 8: 1398-1406, 2006.

10. Wu ZQ, Li XY, Hu CY, Ford M, Kleer CG and Weiss SJ: Canonical Wnt signaling regulates Slug activity and links epithelial-mesenchymal transition with epigenetic breast cancer 1, early onset (BRCA1) repression. P Natl Acad Sci USA 109: 16654-16659, 2012.

11. Cruciat CM and Niehrs C: Secreted and transmembrane wnt inhibitors and activators. Cold Spring Harb Perspect Biol 5: a015081, 2013.

12. Shimomura Y, Agalliu D, Vonica A, Luria V, Wajid M, Baumer A, Belli S, Petukhova L, Schinzel A, Brivanlou AH, et al: APCDD1 is a novel Wnt inhibitor mutated in hereditary hypotrichosis simplex. Nature 464: 1043-1047, 2010.

13. Takahashi M,Fujita M, Furukawa Y,Hamamoto R, Shimokawa T, Miwa N, Ogawa M and Nakamura Y: Isolation of a novel human gene, APCDD1, as a direct target of the beta-Catenin/T-cel factor 4 complex with probable involvement in colorectal carcinogenesis. Cancer Res 62: 5651-5656, 2002.
14. Mathelier A, Zhao X, Zhang AW, Parcy F, Worsley-Hunt R, Arenillas DJ, Buchman S, Chen CY, Chou A, Ienasescu H, et al: JASPAR 2014: An extensively expanded and updated open-access database of transcription factor binding profiles. Nucleic Acids Res 42 (Database issue): D142-D147, 2014.

15. Messeguer X, Escudero R, Farré D, Núñez O, Martinez J and Albà MM: PROMO: Detection of known transcription regulatory elements using species-tailored searches. Bioinformatics 18: 333-334, 2002.

16. Farré D, Roset R, Huerta M, Adsuara JE, Roselló L, Albà MM and Messeguer X: Identification of patterns in biological sequences at the ALGGEN server: PROMO and MALGEN. Nucleic Acids Res 31: 3651-3653, 2003.

17. Lee $\mathrm{C}$ and Huang $\mathrm{CH}$ : LASAGNA-Search: An integrated web tool for transcription factor binding site search and visualization. Biotechniques 54: 141-153, 2013.

18. Livak KJ and Schmittgen TD: Analysis of relative gene expression data using real-time quantitative PCR and the 2(-Delta Delta C(T)) method. Methods 25: 402-408, 2001.

19. Kretschmer C, Sterner-Kock A, Siedentopf F, Schoenegg W, Schlag PM and Kemmner W: Identification of early molecular markers for breast cancer. Mol Cancer 10: 15, 2011.

20. Edgar R, Domrachev M and Lash AE: Gene expression omnibus: NCBI gene expression and hybridization array data repository. Nucleic Acids Res 30: 207-210, 2002.

21. Pawitan Y, Bjöhle J, Amler L, Borg AL, Egyhazi S, Hall P, Han X, Holmberg L, Huang F, Klaar S, et al: Gene expression profiling spares early breast cancer patients from adjuvant therapy: Derived and validated in two population-based cohorts. Breast Cancer Res 7: R953-R964, 2005.

22. Calabrò A, Beissbarth T, Kuner R, Stojanov M, Benner A, Asslaber M, Ploner F, Zatloukal K, Samonigg H, Poustka A and Sültmann H: Effects of infiltrating lymphocytes and estrogen receptor on gene expression and prognosis in breast cancer. Breast Cancer Res Treat 116: 69-77, 2009.

23. Graham TA, Ferkey DM, Mao F, Kimelman D and Xu W: Tcf4 can specifically recognize beta-catenin using alternative conformations. Nat Struct Biol 8: 1048-1052, 2001.

24. Morin PJ, Sparks AB, Korinek V, Barker N, Clevers H, Vogelstein B and Kinzler KW: Activation of beta-catenin-Tcf signaling in colon cancer by mutations in beta-catenin or APC. Science 275: 1787-1790, 1997.

25. Poy F, Lepourcelet M, Shivdasani RA and Eck MJ: Structure of a human Tcf4-beta-catenin complex. Nat Struct Biol 8: 1053-1057, 2001.

26. Matsuda Y, Schlange T, Oakeley EJ, Boulay A and Hynes NE: WNT signaling enhances breast cancer cell motility and blockade of the WNT pathway by sFRP1 suppresses MDA-MB-231 xenograft growth. Breast Cancer Res 11: R32, 2009.

27. Mikheev AM, Mikheeva SA, Maxwell JP, Rivo JV, Rostomily R, Swisshelm K and Zarbl H: Dickkopf-1 mediated tumor suppression in human breast carcinoma cells. Breast Cancer Res Treat 112: 263-273, 2008.

28. Trifa F, Karray-Chouayekh S, Jmal E, Jmaa ZB, Khabir A, Sellami-Boudawara T,FrikhaM,Daoud J and Mokdad-Gargouri R: Loss of WIF-1 and Wnt5a expression is related to aggressiveness of sporadic breast cancer in Tunisian patients. Tumor Biol 34: 1625-1633, 2013.

29. Suzuki H, Toyota M, Caraway H, Gabrielson E, Ohmura T, Fujikane T, Nishikawa N, Sogabe Y, Nojima M, Sonoda T, et al: Frequent epigenetic inactivation of Wnt antagonist genes in breast cancer. Br J Cancer 98: 1147-1156, 2008. 